\title{
Reconstruction of a nonlinear heat transfer law from incomplete boundary data by means of infrared thermography
}

\author{
Fabrizio Clarelli ${ }^{1}$ and Gabriele Inglese ${ }^{2}$ \\ ${ }^{1}$ Department of Pharmacy, UiT University of Norway, Hansine \\ Hansens veg 18, Tromso, Norway. \\ ${ }^{2} \mathrm{CNR}$ - Istituto per le Applicazioni del Calcolo "M.Picone"; Via M. \\ Del Piano 10, 50019 Sesto Fiorentino (FI), Italy.
}

Abstract. Heat exchange between a conducting plate and the environment is described here by means of an unknown nonlinear function $F$ of the temperature $u$. In this paper we construct a method for recovering $F$ by means of polynomial expansion, perturbation theory and the toolbox of thermal inverse problems. We test our method on two examples: In the first one, we heat the plate (initially at $20^{\circ} \mathrm{C}$ ) from one side, read the temperature on the same side and identify the heat exchange law on the opposite side (active thermography); in the second example we measure the temperature of one side of the plate (initially at $1500{ }^{\circ} \mathrm{C}$ ) and study the heat exchange while cooling (passive thermography). 


\section{Introduction}

The present paper deals with heat exchange between a homogeneous conducting plate $\Omega$ and the environment. It is known that there are cases in which linear Newton's law of cooling fails to describe the physics of the problem [1],[13] and [21].

Moreover, classical nonlinear laws (Dulong-Petit, Newton-Stephan laws) "can be applied with confidence over the range of conditions usually found in laboratory calorimetric experiments" [19] but there are natural and industrial circumstances in which the form of the nonlinearity is unknown and requires a specific analysis (see for example [8], [11], [12], [18] and [20]).

We assume that the heat transfer is described here by an unknown nonlinear function $F$ of the temperature $u$. In this paper we construct a method for recovering $F$ by means of a polynomial expansion, perturbation theory and, finally, the typical toolbox of thermal inverse problems including Tikhonov regularization (see also [13], [5], [9] and [10]). Input data consists of a sequence of temperature maps taken on an accessible subset of the external surface of $\Omega$.

In section 2 we describe in detail the direct model and prove the stability of the temperature with respect to the size of the nonlinearities that appear in the boundary conditions.

The inverse problem and our method for identifying $F$ are described in section 3 .

In section 4 we test the method with two different physical simulations. In both cases only one face of the plate is accessible.

In the first one we simulate the heating of the accessible side of $\Omega$ by means of a controlled flux generated by a lamp (active thermography) and assume that the cooling law on the opposite inaccessible side is an unkown nonlinear perturbation of Newton's law. We identify the nonlinear term in the cooling law from a sequence of temperature maps taken on the accessible side. Temperature ranges from $20{ }^{\circ} \mathrm{C}$ to $45{ }^{\circ} \mathrm{C}$.

The second example deals with cooling from high temperature (from $1500{ }^{\circ} \mathrm{C}$ to $500{ }^{\circ} \mathrm{C}$ ) and is taken from [5]. The specimen is not heated (passive thermography). We show a regularized approximation of the unknown function $F$ whose quality is comparable to the reconstructions proposed in [5] though our assumptions are less restrictive.

Finally, we adopt the following notation for function spaces:

$C(\bar{A})$ is the set of real continuous functions defined on the closed set $\bar{A}$

$C^{k}(A)$ is the set of real continuous functions defined on the open set $A$ whose partial derivatives are continuous up to the order $k=1,2$. 


\section{The Direct Model}

We limit ourselves to the $2 \mathrm{D}$ problem in which $\Omega$ is an orthogonal section of the thin plate. More precisely, let $\Omega$ be the open strip $(-L, L) \times(0, a)$ with $L>>a>0$. For each $\tau>0$, we define $D_{\tau}=\Omega \times(0, \tau]$ and $S_{\tau}=\partial \Omega \times(0, \tau]$. Suppose that $\Omega$ represents a metallic specimen with uniform conductivity $\kappa$.

If $u \in C\left(\overline{D_{\tau}}\right) \cap C^{2}\left(D_{\tau}\right)$ the maximum norm of $u$ is defined as $\|u\|_{\infty} \equiv$ $\max _{\overline{D_{\tau}}}|u(x, z, t)|$.

The temperature in $\Omega$ satisfies the heat equation

$$
u_{t}=\alpha \Delta u \quad \text { in } \quad D_{\tau},
$$

with boundary conditions for $x \in(-L, L), t \in(0, \tau]$

$$
\begin{gathered}
u_{z}(x, a, t)+\gamma_{a}\left(u(x, a, t)-U^{a}\right)+\epsilon f(u(x, a, t))=0 \\
-u_{z}(x, 0, t)+\gamma_{0}\left(u(x, 0, t)-U^{0}\right)-\frac{\Phi(x, t)}{\kappa}=0
\end{gathered}
$$

and initial data

$$
u(x, z, 0)=T_{i n}(x, z) \quad \text { for } \quad(x, z) \in \Omega .
$$

On the vertical sides of $\Omega$ we assume the adiabatic conditions

$$
-u_{x}(-L, z)=u_{x}(L, z)=0 .
$$

A list of details about physical parameters and mathematical notation follows:

$\alpha=\frac{\kappa}{\rho c}$ is the diffusivity. The plate $\Omega$ is made of a metal of density $\rho$ and specific heat at constant pressure $c$;

$\kappa \gamma_{a}$ and $\kappa \gamma_{0}$ are the coefficients of surface heat transfer corresponding to $z=a$ and $z=0$ respectively;

$U^{a}$ and $U^{0}$ are the temperatures of the surrounding media (assumed constant) while the initial temperature $T_{i n}$ is a smooth function defined in $\Omega$

$\gamma\left(u(x, a, t)-U^{a}\right)+\epsilon f(u(x, a, t))$ accounts for the nonlinear functional relation between the surface temperature and the rate of heat exchange through the upper side of $S_{\tau}$. The parameter $\epsilon>0$ is the scale factor of the nonlinearity. The function $f$ belongs to $C(J)$. The set $J$ is called "the sector between upper and lower solutions" and will be defined in the next section.

$u^{\epsilon}$ is the solution of the Initial Boundary Problem (1)-(4). This notation points out the dependence of the solution on the scale factor $\epsilon$. Hence, $u^{0}$ 
is the "background" temperature corresponding to the linear heat exchange $u_{z}(x, a, t)+\gamma_{a}\left(u(x, a, t)-U^{a}\right)=0$ for $x \in(-L, L), t \in(0, \tau]$.

$\Phi$ is a prescribed flux into the surface $z=0$. It is generated by a controlled heat source (a lamp, a battery of lamps, a laser). Usually it takes the form $\Phi(x, t)=\Phi_{0}(x) F(t)$ where $\Phi_{0}>0$ and $F(t)$ can be either a periodic function (lock-in thermography) or a pulse (pulsed thermography) [13].

\subsection{Pao's results about the direct model}

The theoretical background of the direct model consists of a set of results by Pao [14] in which existence and uniqueness of solutions of parabolic equations with nonlinear boundary conditions are proven under suitable hypotheses. The main theoretical statement (Theorem 1.1 Chapt.4 [14]) suggests a strategy for the numerical approximation of the solution as limit of a monotone sequence of solutions of linear problems. Stability of the solution of the Direct Model (1)-(4) with respect to $\epsilon$ is a corollary of theorem (Theorem 1.1 Chapt. 4 [14]).

To lighten the notation, in (1)-(4) we set $U^{a}=U^{0}=0$ and $\gamma=\gamma_{0}$. In the introduction, the geometry of our thin plate was described by a rectangular strip of thickness $a>0$. In order to apply Pao's theorem, here we assume that $\Omega$ is the convex open set in $R^{2}$

$$
S_{-} \cup R \cup S_{+}
$$

where:

$$
R=(-L, L) \times(0, a)
$$

with $(a<<L)$,

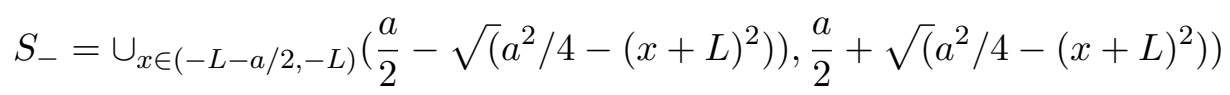

$$
\begin{aligned}
& \left.S_{+}=\cup_{x \in(L, L+a / 2)}\left(\frac{a}{2}-\sqrt{(} a^{2} / 4-(x-L)^{2}\right)\right), \frac{a}{2}+\sqrt{\left.\left(a^{2} / 4-(x-L)^{2}\right)\right) .}
\end{aligned}
$$

The domain $\Omega$ looks like a finite thin strip with smoothed corners. Its boundary is a closed curve of class $C^{1}$.

Furthermore, we write down our boundary conditions in the form

$$
\frac{\partial u}{\partial \nu}(P, t)+\gamma u=g(t, P(x, z), u)
$$

where $P(x, z) \in \partial \Omega$. The function $g$ is continuous and it is piecewise defined as

$$
g(t, P, u)=-\epsilon f(P, u(x, a, t)) \quad \text { in } \quad\{(x, a) \quad \text { with } \quad x \in(-L, L)\}
$$


and

$$
g(t, P, u)=\frac{\Phi(x, t)}{\kappa} \quad \text { in } \quad\{(x, 0) \quad \text { with } \quad x \in(-L, L)\}
$$

with $f(P, u)=\Phi(x, t)=0$ for $L>|x| \geq \frac{9}{10} L$. In the rest of the boundary we have $g=0$.

To describe the essentials of this result, we must introduce the definition of upper (lower) solution: A function $\tilde{u} \in C\left(\bar{D}_{\tau}\right) \cap C^{2}\left(D_{\tau}\right)$ is called an upper solution of (1)-(4) if it satisfies the inequalities

$$
\begin{gathered}
\tilde{u}_{t}-\alpha \Delta \tilde{u} \geq 0 \quad \text { in } \quad \Omega \times(0, \infty) \\
\tilde{u}_{\nu}+\gamma \tilde{u} \geq g \quad \text { on } \quad S_{\tau} .
\end{gathered}
$$

and the initial condition

$$
\tilde{u}(x, z, 0) \geq T_{\text {in }}(x, z) \equiv 0
$$

in $\Omega$.

As for the lower solution $\hat{u}$ the definition is the same, only changing $\geq$ with $\leq$. The sector $J=<\hat{u}, \tilde{u}>$ is defined as

$$
J=\left\{v \in C\left(\bar{D}_{T}\right) \quad \text { s.t. } \quad \hat{u} \leq v \leq \tilde{u}\right\} .
$$

Assume that $g \in C\left(S_{\tau} \times J\right)$ and that $g$ is Lipschitz in $J$.

The existence of a unique solution $u$ of (1)-(4) is proved by Pao in Theorem 4.1.1 [14] under the assumption that there exist a lower and an upper solution $\hat{u}$ and $\tilde{u}$ of (1)-(4). The proof is based on the iterative construction of two sequences, $\left\{\hat{V}^{k}\right\}$ and $\left\{\tilde{V}^{k}\right\}$, that converge monotonically to the solution $u$. The decreasing sequence $\left\{\tilde{V}^{k}\right\}$ starts with the upper solution $\tilde{u}$ and approximates the solution $u$ from behind while $\left\{\hat{V}^{k}\right\}$ starts with the lower solution $\hat{u}$ and converges to $u$ monotonically from below. Numerical implementation of $\left\{\hat{V}^{k}\right\}$ and $\left\{\tilde{V}^{k}\right\}$ seems to be very expensive though each $\tilde{V}^{k}$ is determined by solving a linear BVP whose boundary conditions involve $\tilde{V}^{k-1}$. Details about the definition of sequences $\left\{\hat{V}^{k}\right\}$ and $\left\{\tilde{V}^{k}\right\}$ are in [14] section 4.1. This construction furnishes the main tool for providing the following stability estimate for $u$.

Finally, we show that the sector $J$ is not empty. Actually, consider the linear function

$$
\tilde{u}=C\|\Phi\|_{\infty}+D \epsilon\|f\|_{\infty}-\|\Phi\|_{\infty} z
$$

with $C \geq \frac{1}{\gamma}\left(\frac{1}{\kappa}+1+a\right)$ and $D \geq \frac{1}{\gamma}$. Straightforward calculations show that $\tilde{u}$ is an upper solution and $\hat{u}=-\tilde{u}$ is a lower solution of (1)-(4). 


\subsection{Stability of the direct model with respect to $\epsilon$}

Theorem. Let $u^{\epsilon} \in C\left(\bar{D}_{\tau}\right) \cap C^{2}\left(D_{\tau}\right)$ be the solution of (1)-(4). We have

$$
\left\|u^{\epsilon}-u^{0}\right\|_{\infty} \leq C \epsilon
$$

Proof. We recall that $\left\{\tilde{V}^{k}\right\}$ converges monotonically to $u^{\epsilon}$. It means that

$$
\left\|u-\tilde{V}^{k}\right\|_{\infty} \leq C_{1} \epsilon
$$

for $k \geq k 1$. The IBVP solved by $\tilde{V}^{k}$ is linear. If $\tilde{u}^{0 k}$ is an upper solution of (1)-(4) when $\epsilon=0$, it is well known (stability of linear IBVP w.r.t. parameters, see for example [17] pag. 507) that

$$
\left\|\tilde{V}^{k}-\tilde{u}^{0 k}\right\|_{\infty} \leq C_{2} \epsilon .
$$

On the other hand, we have

$$
\left\|u^{0}-\tilde{u}^{0 k}\right\|_{\infty} \leq C_{3} \epsilon
$$

for $k \geq k 2$. Finally, (8) turns out to be true for $C=C_{1}+C_{2}+C_{3}$ and $k \geq \max \{k 1, k 2\}$.

\section{The inverse problem}

The Initial Boundary Value Problem (IBVP) (1)-(4) is the frame (Direct Model) in which we define the following inverse problem:

IP Identify the nonlinear term $\epsilon f$ from the knowledge of a finite sequence of temperature measurements taken on a portion of the boundary of $\Omega$.

A similar problem, posed in the stationary frame of Laplace's equation, has been studied in [6] and [7]. A stability estimates for the solution of IP is given in [15].

\subsection{Approximation of $\tilde{f}$ from perturbative analysis of the direct model}

The nonlinear term $\epsilon f$ in (2) is unknown. We will use the equations (1)-(4)of the direct model and the knowledge of thermal contrast for $z=0$ to recover $\epsilon f$.

First, we plug the formal expansion $u^{\epsilon}=u 0+\epsilon u 1+O\left(\epsilon^{2}\right)$ in the IBVP (1)-(4) and transform it in a perturbative hierarchy of linear problems. We consider only order zero and order one of the scheme. 
The term $u 0$ satisfies the heat equation

$$
u_{t}=\alpha \Delta u \quad \text { in } \quad D_{\tau}
$$

with boundary-initial conditions

$$
\begin{gathered}
-u_{z}(x, 0, t)=\frac{\Phi(x, t)}{\kappa} \quad \text { for } \quad x \in(-L, L) \quad t \in(0, \tau) \\
u_{z}(x, a, t)+\gamma\left(u(x, a, t)-U^{a}\right)=0 \quad \text { for } \quad x \in(-L, L) \quad t \in(0, \tau) \\
u(x, z, 0)=T_{\text {in }}(x, z) \quad \text { for } \quad(x, z) \in \Omega .
\end{gathered}
$$

The solution $u 0$ is just the background temperature $u^{0}$. Observe that (12)(15) is well posed and, in particular, once the physical parameters $T_{i n}, \alpha$, $\gamma, \kappa$ and $\Phi$ are known, $u^{0}$ is uniquely determined. On the other hand, the function $W=\epsilon u 1$ fulfills the heat equation

$$
W_{t}=\alpha \Delta W \quad \text { in } \quad D_{\tau}
$$

with linear boundary conditions

$$
W_{z}(x, a, t)+\gamma W(x, a, t)+\tilde{f}\left(u^{0}\right)=0 \quad x \in(-L, L) \quad t \in(0, \tau)
$$

with $\tilde{f}=\epsilon f$,

$$
W_{z}(x, 0, t)=0 \quad x \in(-L, L) \quad t \in(0, \tau)
$$

and initial conditions

$$
W(x, z, 0)=0 \quad \text { for } \quad(x, z) \in \Omega .
$$

The thermal contrast (measured in real cases by means of an infrared camera)

$$
G(x, t)=u^{\epsilon}(x, 0, t)-u^{0}(x, 0, t) \quad x \in(-L, L) \quad t \in(0, \tau)
$$

gives us the following noisy additional boundary condition that will be used in section 3.2 to recover $\tilde{f}$ :

$$
W(x, 0, t) \approx G(x, t)
$$

\subsection{The expansion of $u^{\epsilon}$ is not merely formal}

Actually, we prove that $\frac{\left\|u-u^{0}-W\right\|}{\epsilon}=O(\epsilon)$. We set

$$
v=\frac{u-u^{0}-W}{\epsilon} \text {. }
$$


We have

$$
v_{t}=\alpha \Delta v \quad \text { in } \quad D_{\tau}
$$

with boundary and initial conditions

$$
\begin{gathered}
v_{z}(x, a, t)+\gamma v(x, a, t)-\left(f(u(x, a, t))-f\left(u^{0}(x, a, t)\right)\right)=0 \quad x \in(-L, L) \\
\quad t \in(0, \tau) \\
v_{z}(x, 0, t)=0 \quad x \in(-L, L) \quad t \in(0, \tau)
\end{gathered}
$$

and

$$
v(x, z, 0)=0 \quad \text { for } \quad(x, z) \in \Omega .
$$

Since $f \in C(J)$ is supposed Lipschitz in $J$, we have

$$
\left\|f(u)-f\left(u^{0}\right)\right\| \leq\|f\|_{\infty}\left\|u-u^{0}\right\| \leq C\|f\|_{\infty} \epsilon .
$$

It comes from classical estimates (see [17] page 507) that

$$
\|v\| \leq A \epsilon
$$

\subsection{Discretization and approximation of $\tilde{f}$}

Let $\left\{\Psi_{k}: J \rightarrow \mathbb{R}\right\}_{k=0}^{\infty}$ be a sequence of linearly independent functions that span $C(J)$ so that $f(v)=\sum_{k} \beta_{k} \Psi_{k}(v)$. Furthermore, let $W^{(k)}$ solve the linear IBVP

$$
W_{t}=\alpha \Delta W \quad \text { in } \quad D_{\tau}
$$

with boundary conditions

$$
\begin{gathered}
W_{z}(x, 0, t)=0 \\
W_{z}(x, a, t)+\gamma W(x, a, t)-\Psi_{k}\left(u^{0}\right)=0
\end{gathered}
$$

and initial data

$$
W(x, z, 0)=0 .
$$

It comes from linearity that the solution of (16)-(19) takes the form of

$$
W(x, z, t ; \beta)=\sum_{k} \beta_{k} W^{(k)}\left(u^{0}(x, z, t)\right) .
$$

Since $\tilde{f}$ (or, equivalently, the vector $\beta$ ) is not known, we can try to approximate it from the knowledge of the thermal contrast $G(x, t)=u^{\epsilon}(x, 0, t)-$ 
$u^{0}(x, 0, t)$. To do this, we set the following minimum problem in finite dimension

$$
\min _{\beta=\left(\beta_{0}, \ldots, \beta_{N}\right)}\left\{\|W(x, 0, t ; \beta)-G(x, t)\|_{2}^{2}+\lambda B(\beta)\right\} .
$$

Since the $W^{(k)}$ s are linearly independent, any finite Gram matrix $H^{(N)}=$ $\left(\int_{[-L, L] \times[0, \tau]} W^{(j)} W^{(k)} d x d t\right)_{j, k=0, \ldots, N}$ is expected to be nonsingular, even if it could be severely ill-conditioned. For this reason, regularization is required to handle truncation errors and the effects of noise affecting our data.

As for the penalty $B(\beta)$, it must be chosen using a priori information if available. In fact, we assume that $\tilde{f}$ is smooth and increasing. This assumption is supported by a number of known examples of nonlinear heat transfer coefficients ([1], [5], [12], [4] and many others) and by private discussions. The idea is that the higher the temperature, the greater the rate at which heat passes through an interface. Moreover, sudden jumps related to the temperature increase are not expected. A good choice of $B$ could be the $L^{2}$ norm of the first derivative of $\tilde{f}$.

Hence, we assume that $\tilde{f}(\xi)(\xi \in J)$ is a non decreasing function which can be approximated by means of low order polynomials. At this stage, we assume $\Psi_{k}(\xi)=\xi^{k}$ so that we will work with the finite expansion

$$
\tilde{f}^{(N)}(\xi)=\sum_{k=0}^{N} \beta_{k} \xi^{k}
$$

In our tests, it will be $N=4$. This value of $N$ comes from a compromise between the accuracy of the approximation and the stability of the solutions. We write the $L^{2}$ norm of $\tilde{f}^{\prime}(\xi)=\sum_{k=1}^{N} k \beta_{k} \xi^{k-1}$ as the quadratic form in $\beta$

$$
\left(B^{(N)} \beta, \beta\right)=\sum_{k, j=1}^{N} k \beta_{k} j \beta_{j} \int_{[-L, L] \times[0, \tau]} u^{0}(x, 0, t)^{j+k-2} d x d t .
$$

Finally, the Euler equations of our minimum problem are

$$
\begin{gathered}
\sum_{k=0}^{N}\left(\int_{[-L, L] \times[0, \tau]} W^{(j)} W^{(k)} d x d t+\lambda k j \int_{[-L, L] \times[0, \tau]} u^{0}(x, 0, t)^{j+k-2} d x d t\right) \beta_{k}= \\
\int_{[-L, L] \times[0, \tau]} W^{(j)} G d x d y d t
\end{gathered}
$$

for $j=0, \ldots, N$. 


\section{$4 \quad$ Numerical tests}

We apply the perturbative scheme introduced in section 3.1 to the reconstruction of unknown nonlinear heat exchange laws in two different examples:

(1) a metallic plate is warmed up starting from $20^{\circ} \mathrm{C}$;

(2) a purely theoretical sample is allowed to cool starting from a temperature of $1500{ }^{\circ} \mathrm{C}$.

\subsection{Active thermography}

We suppose that the heat flux $\Phi$ is constant in $x$ and that the unknown nonlinear function is $\tilde{f}=\epsilon \cdot \eta \cdot\left(u(a, t)-T_{a}\right)^{2}$ with $\epsilon=0.001$ and $\eta=100$. Hence, it is natural to reduce ourselves to a one-dimensional problem (see also [3]) where $z \in[0, a]$. We provide heat to the boundary $z=0$ through a flux of density $\Phi(t)=Q e^{-\frac{t}{\theta}}$ while non-linear heat exchange takes place on the opposite boundary $z=a$.

The temperature of our one-dimensional sample is a function $u^{\epsilon}(z, t)$ that solves the Initial Boundary Value Problem

$$
\begin{gathered}
u_{t}=\alpha u_{z z}, \\
u_{z}(0, t)=\gamma_{0} \cdot\left(u(0, t)-T_{a}\right)-\frac{Q}{\kappa} e^{-\frac{t}{\theta}}, \\
u_{z}(a, t)=-\gamma_{a} \cdot\left(u(a, t)-T_{a}\right)-\epsilon \cdot \eta \cdot\left(u(a, t)-T_{a}\right)^{2}, \\
u(z, 0)=T_{a} ;
\end{gathered}
$$

where $\alpha=6 \cdot 10^{-5} \mathrm{sec} / \mathrm{m}^{2}, T_{a}=20{ }^{\circ} \mathrm{C}, \gamma=10 \mathrm{~m}^{-1}, \frac{Q}{\kappa}=10000{ }^{\circ} \mathrm{C} / \mathrm{m}$, $\theta=1$ sec.

We solve numerically equations (33), (34), (35) and (36) by means of a finite difference scheme (for the explicit and imex numerical schemes adopted here, see [2]). The trace $u^{\epsilon}(0, t)$ of the solution is assumed to play the role of real temperature data. In experiments, these values are taken by means of an infrared camera.

$u^{0}(x, t)$ is the background temperature corresponding to $\epsilon=0$ i.e. the situation in which heat exchange through the boundary $z=a$ follows Newton's cooling law.

Consider the expansion $u^{\epsilon}=u^{0}+\epsilon u 1+O\left(\epsilon^{2}\right)$. It is easy to check that $\epsilon u 1(0, t)+O\left(\epsilon^{2}\right)$ is just the thermal contrast considered in the definition 
of the inverse problem IP. Since $\epsilon$ is actually not known, we set $W=\epsilon u 1$ and $\tilde{\eta}=\epsilon \eta$. We plug this expansion in the IBVP above and carry out a perturbative analysis.

\subsection{1 order zero}

The function $u^{0}$ fulfills

$$
\begin{gathered}
u_{t}=\alpha u_{z z} . \\
u_{z}(0, t)=\gamma \cdot\left(u(0, t)-T_{a}\right)-\frac{Q}{\kappa} e^{-t}, \\
u_{z}(a, t)=-\gamma \cdot\left(u(a, t)-T_{a}\right) .
\end{gathered}
$$

Because of the hierarchic structure of perturbations, $u^{0}$ will enter as a given quantity in the following IBVP corresponding to the order one.

\subsection{2 order one}

The scaled first order solution $W$ solves

$$
\begin{gathered}
W_{t}=\alpha W_{z z}, \\
W_{z}(0, t)=\gamma W(0, t), \\
W_{z}(a, t)=-\gamma \cdot W(a, t)-\tilde{\eta}\left(u^{0}(a, t)-T_{a}\right)^{2}, \\
W(z, 0)=0 .
\end{gathered}
$$

It comes from the linearity of (40)-(43) that $W(z, t)=\sum_{k=0}^{N} \beta(k) W^{(k)}(z, t)$ where $W^{(k)}$ is the solution of

$$
\begin{gathered}
W_{t}=\alpha W_{z z}, \\
W_{z}(0, t)=\gamma \cdot W(0, t), \\
W_{z}(a, t)=-\gamma \cdot W(a, t)-\left(u^{0}(a, t)-T_{a}\right)^{k}, \\
W(z, 0)=0
\end{gathered}
$$

for $k=0, . ., N$.

We recall that $W(0, t)$ is the thermal contrast defined in $(20)$. To make contrast data more realistic, we add white gaussian noise $g_{\sigma}(t)$ of average zero and standard deviation $\sigma=0.3{ }^{\circ} \mathrm{C}$.

Since the matrix $\int_{0}^{\tau} W^{(j)}(t) W^{(k)}(t) d t$ is ill-conditioned, to determine the values of $\beta(0), \ldots, \beta(4)$ we solve the linear system (32) for $N=4$ with an 
optimal choice of the regularization parameter $\lambda$. Recall that the penalty function $B$ is the euclidean norm of the first derivative of the nonlinear term $f$.

The parameter $\lambda$ is determined by means of Hansen's L-curve method [9]. In figures 1 , we summarize the essentials of the numerical example:

Figure 1a. We plot the graphs of $U(t)=u^{\epsilon}(0, t)+g_{\sigma}(t)$ (that plays the role of experimental temperature data at the boundary $z=0)$ and $u^{0}(0, t)$ (the solution of order zero).

Figure 1b. We plot the curve $\left(\left\|\sum \beta_{k}(\lambda) W^{(k)}-U\right\|_{2}^{2},\|B(\lambda)\|_{2}^{2}\right)$ parametrized by $\lambda$. The corner suggests a choice for the numerical value of $\lambda$.

Figure 1c. Finally, we compare the unknown $\tilde{\eta}$ with the polynomial reconstructions with $\lambda=0$ and $\lambda_{\text {opt }}=1.4 \cdot 10^{-10}$.

\subsection{Cooling}

We simulate cooling of a one-dimensional specimen represented by the interval $(0, a)$ by means of the following IBVP (see [5]):

$$
u_{t}=10^{-6} u_{z z} \quad \text { in } \quad D_{\tau}
$$

where $D_{\tau}=(0, a) \times(0, \tau)$,

$$
\begin{gathered}
u_{z}(0, t)=0, \\
u_{z}(a, t)+F(u)=0, \\
u(z, 0)=1500{ }^{\circ} C .
\end{gathered}
$$

Let us assume

$$
F(u)=\frac{A}{2000-u}(u-500),
$$

where $A=367500$. We recall that there is not controlled heat flux here $(\Phi=0)$.

The function $T(t)=u(a, t)$ simulates the collection of data by means of the infrared camera. Simulated temperature for $z=a$ become more realistic by adding for all $t \in(0, \tau)$ a random variable $g$ with uniform distribution on the interval $\left[-5^{\circ} C, 5^{\circ} C\right]$ (the same noise used in [5]).

In order to apply again the perturbative scheme of the previous example we introduce a linear part $\gamma_{0}(u-q)$ so that $\tilde{f}(u)=F(u)-\gamma_{0}(u-q)$. A natural choice for $q$ is the surrounding temperature $q=500{ }^{\circ} C$. We leave $\gamma_{0}$ as a free parameter that will be identified in the next subsection. 


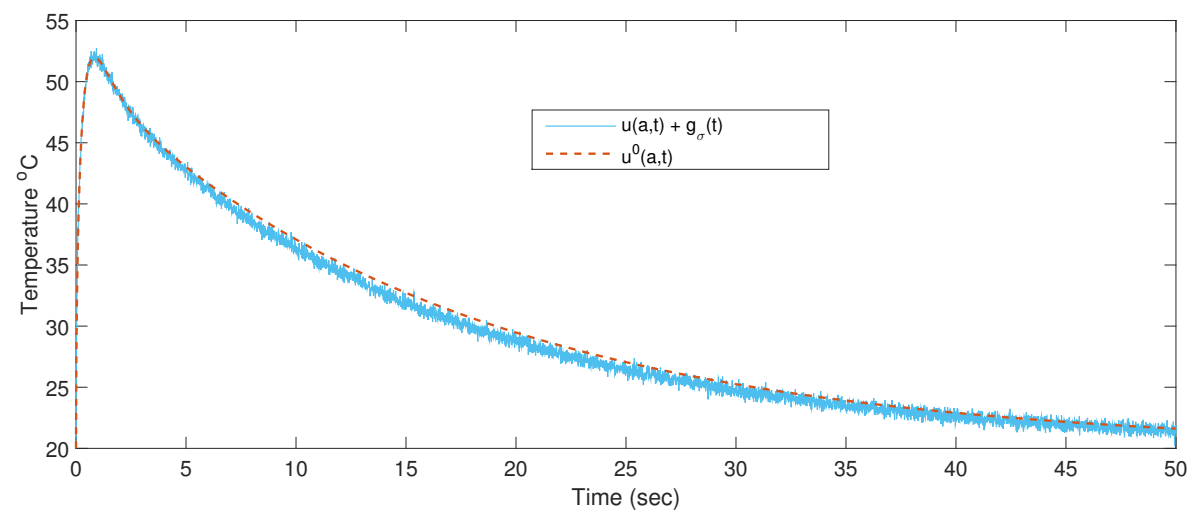

(a) Comparison between temperature data with noise and order zero solution.

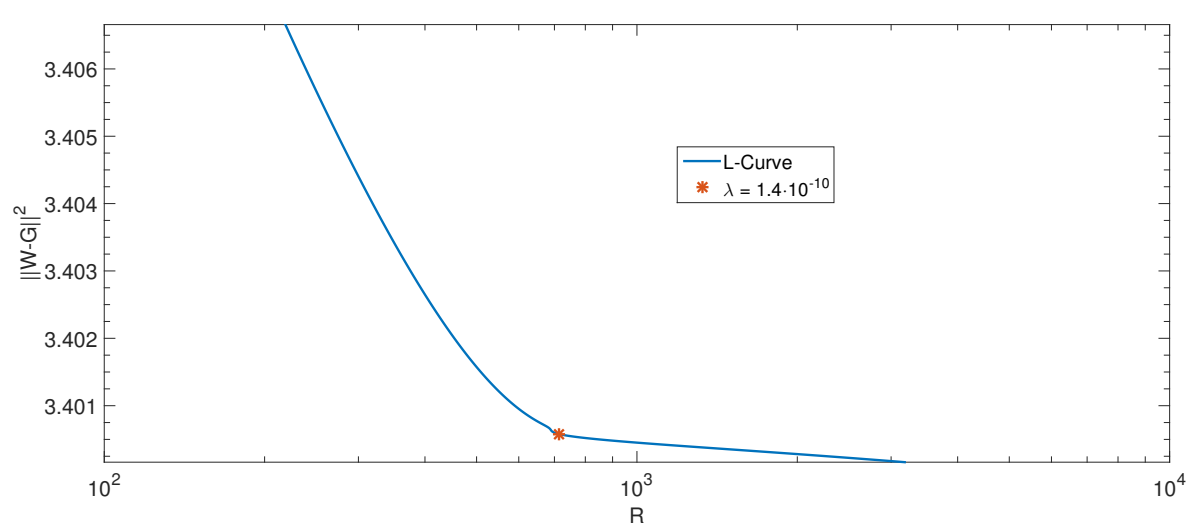

(b) The L-curve helps to choose a value for $\lambda$.

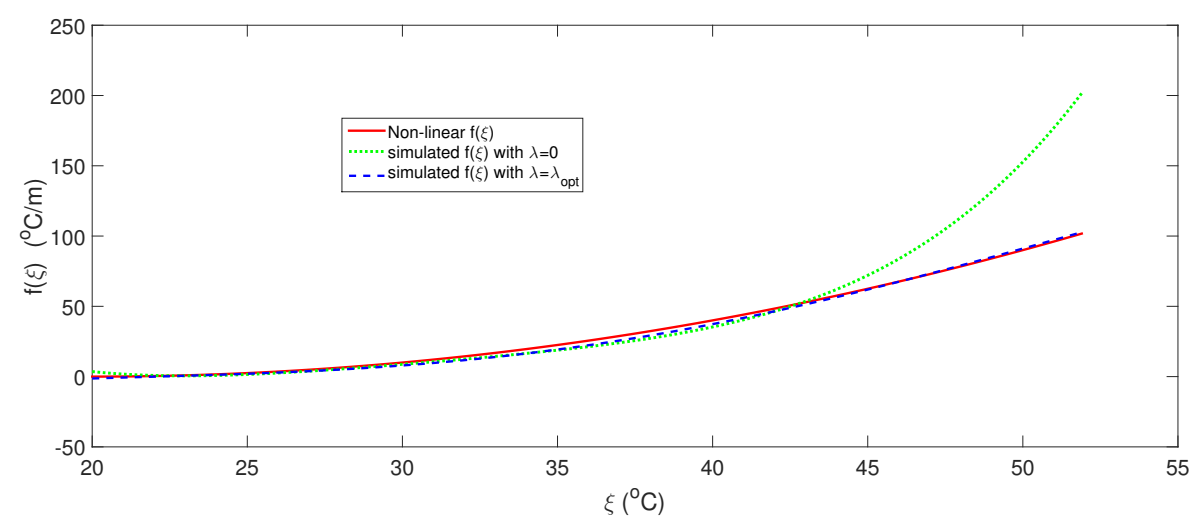

(c) Comparison between the unknown nonlinearity and its approximation with $\lambda=0$ (no regularization) and $\lambda=\lambda_{\text {opt }}$ (from L-curve).

Figure 1: Recovering a small nonlinear term with active thermograpphy. 


\subsubsection{Order zero. Choice of the linear part.}

The order zero solution $u^{0, \gamma_{0}}$ (we are stressing the dependence on $\gamma_{0}$ ) solves the IBVP

$$
\begin{gathered}
u_{t}=10^{-6} u_{z z} \\
u_{z}(0, t)=0 \\
u_{z}(a, t)+\gamma_{0}(u(a, t)-500)=0 .
\end{gathered}
$$

The parameter $\gamma_{0}$ must be chosen to minimize the integral distance $\int_{1000}^{1500}\left|u^{0, \gamma_{0}}(a, t)-T(t)\right|^{2} d t$. The best value is $\gamma_{0} \approx 443 \mathrm{~m}^{-1}$.

\subsection{2 order one}

We compute a finite basis $\left\{W^{(k)}\right\}$ solving the IBVP

$$
\begin{gathered}
W_{t}=10^{-6} W_{z z} \quad \text { in } \quad D_{\tau} \\
W_{z}(0, t)=0, \\
W_{z}(a, t)+\gamma_{0} W+\left(u^{0}(a, t)-500\right)^{k}=0 .
\end{gathered}
$$

for $k=0, . ., 4$. Then, using eqs. (31) and (32), we estimate the vector coefficient $\beta$ to approximate the nonlinear part $\tilde{f}(u)=F(u)-\gamma_{0}(u-500)$.

Remark. We observe numerically that the temperature approximation of the first order $u^{0}(a, t)+W(a, t)$ is a very good approximation of the solution of (48)-(51) (see Figure 2).

Since the matrix $\int_{0}^{\tau} W^{(j)}(t) W^{(k)}(t) d t$ is severely ill-conditioned, the values of $\beta(0), \ldots, \beta(0)$ are obtained solving the regularized linear system (32)for $N=4$ ). The regularization parameter $\lambda$ is determined by means of Hansen's L-curve method [9].

In figures 3, we summarize the essentials of the numerical example:

Figure 3a. Here, we plot the functions $u(a, t)+g(t)$ (that plays the role of experimental temperaure data at the boundary $z=0$ ) and $u^{0}(0, t)$ (the solution of order zero).

Figure 3b. We plot of the curve

$$
\left\|\sum \beta_{k}(\lambda) W^{(k)}-U\right\|_{2}^{2},\|B(\lambda)\|_{2}^{2}
$$

parametrized by $\lambda$. The corner suggests the choice for $\lambda$.

Figure 3c. We compare the unknown $F(u)$ with the polynomial reconstructions with $\lambda=0$ and $\lambda_{\text {opt }}=3.3 \cdot 10^{-16}$. 


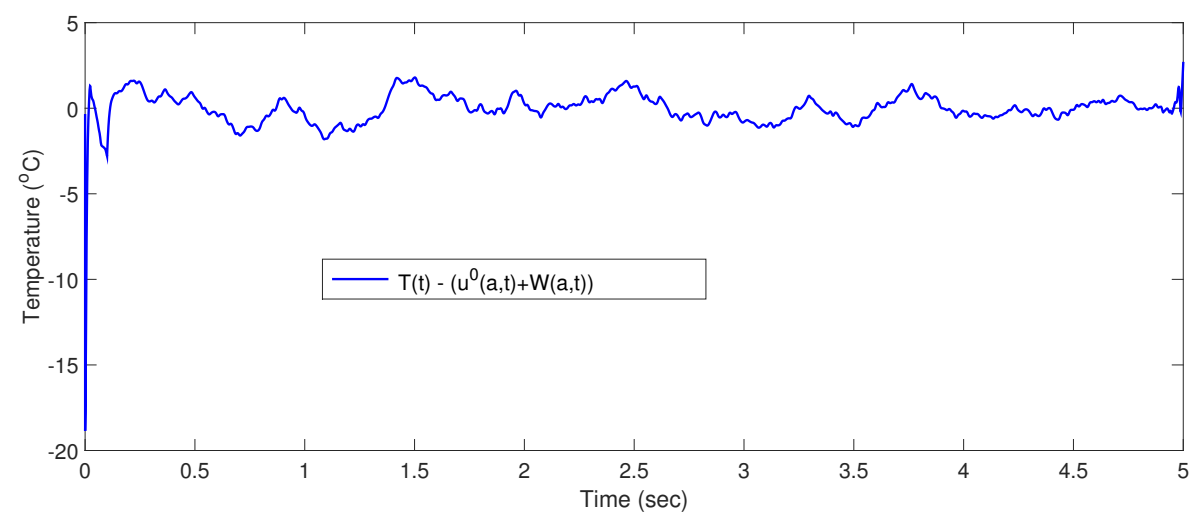

Figure 2: Comparison between $T(t)$ and its first order approximation $u^{0}(a, t)+W(a, t)$.

Finally, the regularized curve shown in Figure 3c is a monotone approximation of $F(u)$ obtained by smoothing the input data and regularizing the solutions. In [5], the additional condition

$$
F(T(0.005))=\frac{A}{2000-u(0.005)}(u(0.005)-500)
$$

is used to obtain a monotone approximation of $F$. In our approximation, we don't use any assumption on the values of $F$.

\section{Conclusions}

We use active infrared thermography and perturbation theory in order to recover an additive nonlinear term in Newton's cooling law. Theoretical background in nonlinear boundary value problems for parabolic equations gives us a stability estimate for the direct model. As for the constructive procedure, we remove non-linearity by means of perturbation theory and produce a regularized polynomial approximation by means of least squares minimization. At present, our algorithm are working with synthetic data.

\section{Acknowledgments}

We wish to thank Dr. Paolo Bison for fruitful discussions, and the referees for their precious suggestions and comments. 


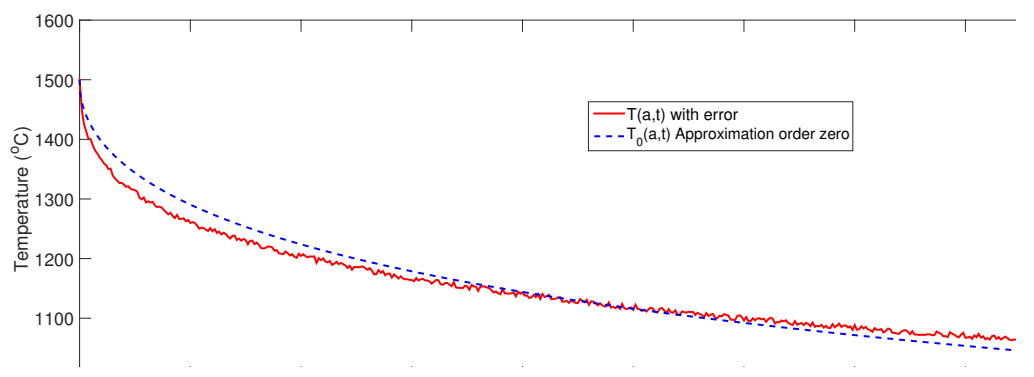

(a) Comparison between $T(t)$ with error and $T_{0}$.

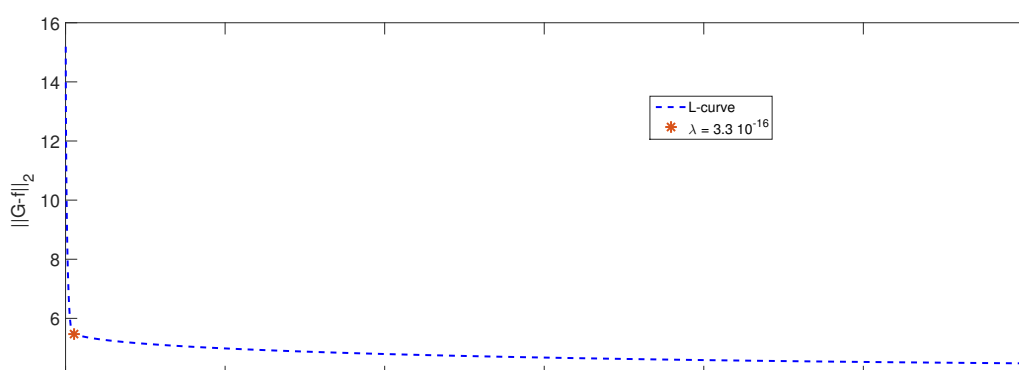

(b) The L-curve helps to choose the regularization parameter $\lambda$.

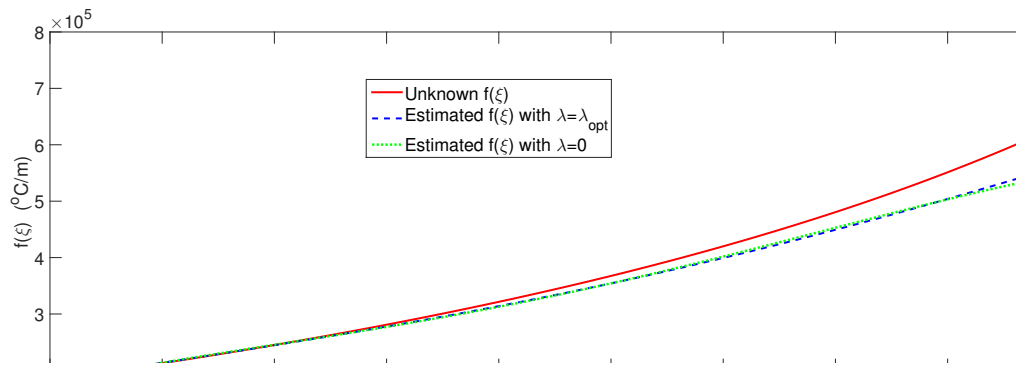

(c) Comparison between the unknown nonlinearity and its approximation with $\lambda=0$ (no regularization) and $\lambda=\lambda_{\text {opt }}$ (from L-curve).

Figure 3: Reconstruction of a nonlinear cooling law at high temperature. 


\section{References}

[1] Carslaw H S and Jaeger J C 1959 Conduction of Heat in Solids (Oxford: Clarendon Press)

[2] Clarelli F., Natalini R., A pressure model of immune response to mycobacterium tuberculosis infection in several space dimensions, Math Biosci Eng., (2010), 7(2), 277-300.

[3] Hao D N, Huong B V, Thanh P X and Lesnic D Identification of nonlinear heat transfer laws from boundary observations Applicable Analysis available online

[4] A. Dehghani, A. Moradi, M. Dehghani, A.Ahani Nonlinear Solution for Radiation Boundary Condition of Heat Transfer Process in Human Eye . 33rd Annual International Conference of the IEEE EMBS Boston, Massachusetts USA, August 30 - September 3, 2011

[5] H. W. Engl, P. Fusek and S. V. Pereverezev, Natural linearizationfor the identification of nonlinear heat transfer laws. J. Inv. Ill-Posed Problems (2005) 13(6) 567-582.

[6] Fasino D and Inglese G, (2006) Recovering unknown terms in a nonlinear boundary condition for Laplace's equation IMA J Appl Math 71 (6), 832-852.

[7] Fasino D and Inglese G, (2007) Recovering nonlinear terms in an inverse boundary value problem for Laplace's equation: A stability estimate. JCAM 198 460-470.

[8] W. Grever A Nonlinear Parabolic Initial Boundary Value Problem Modelling the Continuous Casting of Steel, ZAMM 78 (1998) 2, 109-119.

[9] Hansen P C Discrete Inverese Problems. Insight and algorithms. SIAM, Philadelphia 2010.

[10] Inglese $\mathrm{G}$ Recovering the functional form of nonlinear heat transfer by means of thermal imaging, in Engineering Optimization IV, Rodrigues et al (Eds), (Taylor and Francis) 2015.

[11] Lowell R P and Burnell D K 1990 Mathematical modeling of conductive heat transfer froma freezing, convecting magma chamber to a single-pass hydrothermal system. Earth and planetary science letters 104 59-69.

[12] Lentes F T, Siedow N 1999 Three-dimensional Radiative Heat Transfer in Glass Cooling Processes Glastech.Ber.Glass.Sci.Technol. 72(6) 188196. 
[13] Maldague X P V 2001 Theory and Paractice of Infrared Technology for Nondestructive Testing (New York: Wiley and Sons)

[14] Pao C V 1992 Nonlinear Parabolic end Elliptic Equations, (New York: Plenum Press)

[15] A. Rösh, Stability estimates for the identification of nonlinear heat transfer laws. Inverse Problems (1996) 12, 743-756.

[16] A. Rösh, A Gauss-Newton method for the identification of nonlinear heat transfer laws. International Series of Numer. Math. (2001) 139 217-230.

[17] Salsa S (2008) Partial Differential Equations in Action (Milano: Springer)

[18] F.Santosa, M.Vogelius, J.M.Xu. An effective nonlinear boundary condition for a corroding surface. $Z$ angew Math Phys 49, 656-679 (1998)

[19] O'Sullivan C T Newton's law of cooling - A critical assessment Am.J.Phys. 58(10) 956-960 (1990).

[20] E.Tarnow, When Newton's cooling law doesn't work. Am. J. Phys. 62(1) (1994) 89-90.

[21] Vollmer M Newton's law of cooling revisited European Journal of Physics 30 (2009) 1063-1084. 